Rabaska

Revue d'ethnologie de l'Amérique française

\title{
Les voies/voix de la tradition : faire du neuf avec du vieux
}

\section{Donald Deschênes}

Numéro 1, 2003

URI : https://id.erudit.org/iderudit/201611ar

DOI : https://doi.org/10.7202/201611ar

Aller au sommaire du numéro

Éditeur(s)

Société québécoise d'ethnologie

ISSN

1703-7433 (imprimé)

1916-7350 (numérique)

Découvrir la revue

Citer ce document

Deschênes, D. (2003). Les voies/voix de la tradition : faire du neuf avec du vieux. Rabaska, (1), 137-154. https://doi.org/10.7202/201611ar d'utilisation que vous pouvez consulter en ligne.

https://apropos.erudit.org/fr/usagers/politique-dutilisation/ 


\section{Table ronde}

\section{Les voies/voix de la tradition : faire du neuf avec du vieux}

DONALD DESCHÊNES

Société québécoise d'ethnologie, Québec

\section{Présentation}

Depuis trente ans, la tradition de chanson, musique, danse et conte a changé de voix. Si les premiers folkloristes mettaient toute leur énergie à sauvegarder notre patrimoine oral, si les premiers acteurs de la scène musicale s'inspiraient des folk-singers américains, le Québec et la francophonie ont trouvé leurs voix et leurs voies. À la veille du soixantième anniversaire des Archives de folklore, au moment où on assiste à une relève de la garde chez les folkloristes et ethnologues, l'occasion était belle de porter un regard sur le chemin parcouru ces dernières années. Sont-ce toujours les mêmes préoccupations, les mêmes besoins d'identité et d'intégration ? Lors du $12^{\mathrm{e}}$ Festival international des arts traditionnels de Québec (FIAT), dont le thème était Les voies/voix de la tradition, la Société québécoise d'ethnologie (SQE), en collaboration avec le Centre de valorisation du patrimoine vivant (CvPv), anima une table ronde, le vendredi 11 octobre 2002, à la chapelle du Musée de l'Amérique française.

Festival oblige et le sujet étant si vaste, nous avons proposé que la discussion se fasse avec des chercheurs, des collecteurs, des animateurs et des musiciens, des acteurs qui ont participé à cette effervescence, à cette quête de sens. Pour amorcer et stimuler la discussion, nous avons choisi de présenter le premier film de la série Le Son des Français d'Amérique, La révolution $d u$ dansage réalisé en 1976 par André Gladu et Michel Brault, avec la participation de Mme Georgiana Audet et des gens de l'Île d'Orléans, auquel a participé Normand Legault en tant que recherchiste. Ce court métrage d'une trentaine de minutes s'est imposé de lui-même tant par sa proximité géographique que par le thème qu'il développe, soit l'urgence de sauvegarder et l'action que cette sauvegarde impose. Voici la teneur des propos qu'ont échangés les acteurs et les observateurs de cette scène musicale, ceux qui déploient toujours énergie et talent à faire du neuf avec du vieux. 


\section{TABLE RONDE}

Donald Deschênes. Je vois que le film a suscité beaucoup de réactions et de commentaires. J'en ai vu plusieurs réagir, prendre des notes. Cette année, le thème du FIAT est « les voies, v-o-i-e-s, et les voix, v-o-i-x, de la tradition ». Et c'est sur cette question que nous voulons réfléchir. $\mathrm{Au}$ visionnement de ce film, on sent chez madame Audet une urgence. Si je nous replace à cette époque, il y a près de trente ans, au moment où Robert, Normand, Pierre peut-être et moi, Monique et Tess plus tard, nous sommes passés par les classes de Jean Du Berger, il y avait une urgence de recueillir les choses, de collecter pour ne pas, comme madame Audet le dit si bien, que ça se perde. Ici, on le voit au niveau de la danse, Normand et Pierre ont fait à cette époque de nombreuses enquêtes et recherches.

Dans les années 1960, des groupes populaires comme Les Cailloux calquent leur voix sur le Kingston Trio et Raoul Roy se situe dans la tradition des folk-singers américains. Avec les années 1970, une voix bien québécoise émerge avec des groupes comme La Bottine Souriante, Le Rêve du Diable et plusieurs autres. C'est une voix à laquelle plusieurs d'entre vous avez participé d'une façon ou d'une autre.

Pour cette discussion, j'ai rassemblé autour de la table des gens qui ont joué, chacun à sa façon, un rôle actif dans cette évolution. Ce sont RoBert Bouthillier, qui, d'ethnologue et collecteur, est devenu, au fil des ans, notamment en Bretagne, un animateur, un acteur du milieu, de même qu'un chanteur et un conteur : un gars de terrain, en France et au Québec ; Pierre Chartrand, directeur du Centre Mnémo, coordonnateur de Danse-Cadence, enseignant au programme de musique traditionnelle au Cégep de Joliette, spécialisé en danse traditionnelle québécoise et en danse ancienne, et animateur; Monique JuTRAs, qui a fait une thèse de maîtrise sur les chansons traditionnelles à caractère épique et tragique, et chanteuse qui a marché sur les traces d'un Raoul Roy; NoRMAND LEGAULT, qui a fait de la recherche sur la danse au Québec et de l'animation d'un bout à l'autre du pays et en France ; FranCINE REEVES, qui a fait de l'animation de danse en France et qui mène également une recherche sur la chanson ; DANIELle MARTINEAU, musicienne et chercheuse, qui a mené des collectes dans Lanaudière ; et Tess LEBLANC, ethnologue et chanteuse qui travaille en français, en anglais et en gaélique en une espèce de fusion des trois cultures qui l'habitent. Vous entendrez aussi Louis WaUTHIER, de la troupe Les Farandoles de Chicoutimi et metteur en scène du spectacle La Fabuleuse Histoire d'un Royaume, et LisAn HuberT, du groupe Les Crapaudes, qui a fait de la collecte avec Danielle Martineau. 
Enfin, j'ai demandé à JEAN Du BERGER, professeur de l'université Laval à la retraite, d'écouter attentivement et, à la fin, de faire une synthèse.

À qui j'offre d'ouvrir le bal ? Normand Legault que nous voyons dans le film et auquel il a collaboré

NoRMAND LEGAULT. Juste pour ouvrir la discussion, après quinze, vingt ans, je trouve que c'est plaisant de regarder ce film parce que Brault a réussi quand même à aller chercher un peu l'essentiel de ce que tout le monde essaie de représenter. Il a pris un moyen, le cinéma, pour livrer une bonne représentation de la sphère complète qui représente un peu le sacré de la tradition. On voit les éléments importants : la musique, la danse, la chanson, la bouffe, la vie de la communauté, la salle, le discours, les liens entre tout ça. Puis chacun, dans sa démarche personnelle, essaie d'atteindre ce niveau-là. Un film, c'est bon parce qu'on a le vișuel, le sonore, le contenu, ça nous fait embarquer dedans, sauf que, quand, nous, on agit sur scène, peu importe le lieu, il y a toujours cette dynamique-là qu'on essaie de redonner et de transmettre. C'est essentiel pour la compréhension du phénomène de la tradition, de respecter cette sphère, cet espace sacré : le lieu de la tradition. C'est quelque chose de sacré qu'il faut protéger, qu'on peut utiliser toujours en faisant attention de garder ses limites dans l'espèce de sphère, ne pas l'isoler; parce qu'un élément isolé de la tradition devient anonyme. Je pense que Michel Brault réussit à rassembler tout ce qu'il peut y avoir d'essentiel à la conservation de cette sphère. Pour ma part, dans les démarches, les interventions que j'ai à faire, sur scène ou autrement, je situe toujours le contexte. C'est l'identité, notre façon d'être. On l'atteint très bien, dans le film, cette compréhension-là.

PERSONNE DU PUBLIC. J'aurais une question à vous poser. Aujourd'hui, trente ans plus tard, ce ne serait pas en perdition au Québec ?

Normand Legault. Non, pas du tout, parce que ça se transmet d'une autre manière, les jeunes reprennent ça à leur façon, dans le contexte où ils vivent, comme à chaque époque. Sauf que ça se vit avec les moyens d'aujourd'hui. On est transmetteur, toujours un acteur dans cette traditionlà, un jour ou l'autre, dans notre façon de l'utiliser.

Donald DESChÊNEs. Le sacré, la communauté. Robert a des choses à dire à ce sujet. Ou veut-il nous entraîner sur une autre piste ?

RoBert BOUthillier. Non, pas vraiment. En fait, si on parle du film, on va se rendre compte qu'effectivement on est au cœur de plein de choses. Ce que Normand appelle sacré, moi, je l'ai vécu comme une espèce de plongée dans une convivialité. Plus la convivialité que le sacré, sachant que, à travers toutes les expressions traditionnelles quelles qu'elles soient, 
musicales, dansées, chantées, il y a l'aspect formel extérieur, il y a l'enveloppe puis il y a la matière elle-même; puis il y a ce que crée, ce que génère dans une société ou, à ce niveau-ci, une communauté. On avait effectivement l'impression d'être quelque part, qui était un noyau communautaire, le Château Bélair, où les gens se retrouvaient. On sentait qu'il y avait un plaisir collectif de se retrouver régulièrement, là. Il y avait une connivence entre les gens. Je comprends que Normand utilise le mot sacré. Moi, je ne le sentais pas tout à fait de la même façon. Je pense que c'est une question d'étiquette ou de mot qu'on peut apposer sur ces choses-là.

Ce qui m'a frappé, ce sont les voies ou les modes de la transmission. Tout le monde a entendu ce discours-là : j'ai vu le dernier qui savait la dernière danse ou la dernière chanson puis, après lui, personne ne savait rien. On a tous entendu ça puis on s'est tous aperçu que c'était faux. C'est amusant parce que madame Audet s'applique ça à elle-même. Elle dit : " Je suis la dernière ». Alors, non seulement c'est le chercheur qui trouve le dernier des Mohicans, mais c'est le dernier des Mohicans qui dit : je suis le dernier des Mohicans. Or, l'expérience nous montre que des derniers des Mohicans, il y en a par centaines et par milliers.

Donc, finalement, il y a un paradoxe là-dedans. Ça s'en va-t-y ou ça s'en va-t-y pas ? Est-ce vrai qu'on est le dernier et qu'après moi, c'est terminé ? Je dirais qu'il y a un peu des deux. Ça m'a ramené à des réflexions sur la façon dont une tradition se perpétue. Quand je parle de tradition, je parle surtout de pratiques collectives. On peut être porteur de chansons, par exemple, dans une lignée très intime, familiale, sans que ces choses-là soient vraiment partagées de façon communautaire. Ici, on a tout de même un partage communautaire tout à fait intéressant, en particulier pour la danse. Il est assez frappant de constater que les choses ne sont jamais en continuité pure. Elles sont en cycles de continuité et de rupture : pendant un temps, on va danser; pendant un temps, on ne dansera plus ; pendant un temps, des catalyseurs positifs vont faire qu'on va y revenir. C'est amusant parce que tous les gens qui sont autour de cette table, ont tous quelque part le cul entre deux chaises, entre une démarche de recherche puis une démarche de pratique. On vit dans les deux et, d'une certaine façon, par notre action, on sert de catalyseurs positifs, de trucs qui font reprendre des choses. Sauf que, pour répondre un petit peu à la question que madame posait, on fait reprendre des choses dans des contextes qui ne sont plus tout à fait les mêmes. Alors, ce qui change, ce sont les contextes. La danse peut continuer, comme la chanson, comme le conte. Ce qui a changé, en fait, ce sont les circonstances sociales, collectives, qui font que ça ne va pas se produire ou se reproduire de la 
même façon et dans les mêmes conditions. C'est là la charnière culturelle entre un monde mythique, l'empremier des Acadiens ou, ce que madame Audet disait à propos de son père et la danse, et une actualité réductrice par rapport à ça. Il y a des jeux, des cycles, des pertes, des reprises, grâce à des facteurs, des circonstances, des conditions particulières. En particulier par l'action de personnes comme madame Audet et sur une communauté. C'est sans doute vrai ce qu'elle disait, qu'en 1930, 1940, ça ne dansait sans doute plus. Elle ne l'a pas inventé. À un moment donné, elle est devenue un agent catalyseur qui a fait que ç'a recommencé. Dans nos milieux, nous sommes tous des agents catalyseurs. Les exemples, c'est par centaines qu'on pourrait en trouver : les chansons de Lanaudière, les Fest-noz en Bretagne. La tradition, il ne faut pas la voir comme un fil ininterrompu, définitif, un écheveau qu'on tire puis qui vient tout le temps. C'est plus compliqué que ça. Ça suit des circonvolutions et des chemins de transmission, de perpétuation qui ne sont pas simplistes comme du grand-père au père, au fils, au petit-fils. Ça marche de même, mais ça marche aussi de tout autre façon. C'est ce que j'en retire.

Donald Deschênes. Que de pistes intéressantes. Monique.

Monique Jutras. Je réagis à ce que j'ai entendu. Je trouve qu'aujourd'hui, il y a deux choses complètement différentes : la scène et la tradition. Je pense que le mot tradition, ou le phénomène de la tradition, de la transmission, n'a plus le même sens ; on ne reviendra jamais plus en arrière. Il n'y en aura plus de traditions transmises de génération en génération, de façon très pure. Nous sommes soumis à trop de choses. Quand je vais sur une scène, c'était comme cela il y a trente ans aussi, je ne prétends pas transmettre aux gens quelque chose d'authentique. Je fais autre chose avec la tradition. Je n'ai pas cette vision du sacré qu'a Normand. J'y vais avec mon vécu, ma sensibilité artistique. Je m'accompagne à la guitare ; jamais on n'a fait ça dans la tradition. Je m'accompagne dans un type classique qui n'est pas tiré de la tradition des folk-singers américains. J'ai marché sur les traces de Raoul Roy, sauf que je faisais déjà ça avant de le connaître. C'est ce que je savais faire, j'aimais les chansons de folklore, alors, je les faisais sur scène comme ça.

Aujourd'hui, le folklore est comme la langue ; il évolue. C'est ce que les gens en font. Je regarde ce qui se passe aux veillées de la Société pour la promotion de la danse traditionnelle au Québec (SPDTQ) à Montréal, ou ici à Québec, au Cvpv. On danse, on apprend des danses, on a bien du plaisir. Peut-être que les gens qui viennent nous caller des danses essaient d'être très proches du sacré, de ce qui se faisait dans la 
tradition. Tant mieux! Mais pour moi, ce n'est pas ce qui est important. Comme disait Robert, c'est la convivialité. La même chose quand on se regroupe pour chanter. Tout se peut tout en restant à l'intérieur de certaines limites.

Je voudrais aborder un autre sujet qui me tient beaucoup à cour et sur lequel je réfléchis beaucoup actuellement : c'est tout ce qu'on retrouve au niveau de la scène, chez les artistes qui se spécialisent dans la tradition, c'est ce que j'appelle la fusion. Je trouve que c'est une mode actuellement et ça me fait peur. Ça va avec toute cette histoire de mondialisation, d'échanges des cultures. Je me demande jusqu'à quel point on ne perd pas une certaine identité. Il faut voir qu'il y a toujours eu des mélanges entre les cultures, ça n'a jamais été hermétique. Mais ce qui est né est probablement né de contacts prolongés; alors qu'aujourd'hui, c'est instantané. Un joueur de tam-tam africain se retrouve dans un bar où il y a un gars qui joue du folklore québécois. Ils vont mettre du tam-tam et des jeux de pieds. Des fois, il va naître des disques qui peuvent être très bons. Jusqu'à quel point s'agit-il d'une mode?

Un autre point que je veux soulever, c'est jusqu'à quel point, nous, Québécois, en tant que peuple, comme collectivité, on assume notre propre culture. On a encore honte de notre culture traditionnelle; les gens ne connaissent pas la chanson traditionnelle et ses richesses; ils ne connaissent pas non plus la musique traditionnelle. C'est comme si on avait besoin de la fusionner avec un tam-tam africain, au jazz ou au rock, et d'en faire un ragoût pour mieux la faire passer. On cherche à occulter un peu le folklore à l'état pur. J'admets que, des fois, ça peut être bien fait, intéressant, mais il y a le danger de noyer notre identité.

Pierre Chartrand. C'est sûr qu'on a toujours l'idée d'une tradition pure. Tu as parlé de la tradition pure, Robert a parlé d'un passé mythique. L'un dit que c'est vrai, l'autre que c'est faux. Nous, on a commencé dans les années 1970 et, évidemment, c'est ce qu'on a connu qui est la vérité. C'est un peu ce qui ressort. Prenons des exemples. Monique, tu parles de guitare. Dans les années 1950, beaucoup de groupes ont sorti des disques avec de la guitare et de la batterie. Dans les années 1960, on s'est dit : wô ! On va faire ça sérieusement. On a enlevé les guitares électriques et les batteries. Pourtant, les traditionnels, vingt ans avant, faisaient ça.

Il y a toujours une idéologie qui porte chaque renouveau. Quand Barbeau a fait ses Veillées du bon vieux temps à la bibliothèque SaintSulpice, à Montréal, et son Festival de la chanson et des métiers du terroir au Château Frontenac, il faisait venir des quatuors classiques, des cantatrices comme Loraine Wyman. C'était ni plus ni moins qu'une fusion. 
Il y a quelque temps, j'étais chez la fille d'Ovila Légaré. Vous savez, Ovila Légaré jouait du violon et il s'est coupé le doigt. Il s'est donc mis à caller des danses. Sa fille m'a dit : " Il ne savait pas vraiment de danses, il les créait. » Comme il s'agit d'une pratique, la danse est plus symptomatique que la chanson qui est faite pour l'exécution, pour la scène. La danse est une pratique, créée en bonne partie. C'était du set américain qui n'est jamais entré à l'île d'Orléans. À chaque revival, il y a quelque chose d'emprunté qui s'ajoute. Ce qui fait qu'aujourd'hui, la tradition est souvent le résultat d'une tradition plus ou moins longue avec un renouveau de cette période-là.

Tess LeBlanc. Si on regarde le Cap-Breton, à la fin des années 1980, dans les spectacles écossais, il y avait des jeunes qui jouaient, mais c'était plutôt des gens qu'on connaît comme J. -P. Cormier et Gerry Holland. Après la venue d'Ashley Maclsaac, ensuite de Nathalie McMaster, vers 1998, au même festival, il devait y avoir de dix à quinze jeunes qui jouaient très bien. Il y avait eu un engouement, un renouveau. Ils ne jouaient pas du grunge comme Ashley MacIsaac. Ils ont probablement été inspirés par la scène, par cette possibilité, ce rêve de faire de la scène, de jouer. Ç'a été populaire. Ils avaient appris à jouer avec Jean-Jacques en bas de la route ou dans les cours, d'une façon toute traditionnelle. Selon moi, la question est de savoir où ces jeunes apprennent et puisent leur répertoire. Il y a tout à fait de la place pour tout ça, que ce soit Ashley MacIsaac avec son grunge ou Nathalie McMaster qui joue différents airs de partout.

Donald Deschênes. Finalement, il y a fusion, mais également transmission.

Tess LeBlanc. Absolument ! La question est : où est-ce que la transmission du répertoire se fait, où est-ce qu'ils vont le chercher?

Donald Deschênes. Danielle.

Danielle Martineau. J'ai trouvé plaisant que le film commence avec des petits enfants parce que nous avons fait une collecte dans Lanaudière pour savoir comment se faisait la transmission des traditions. La plupart des gens nous ont dit qu'ils y étaient exposés depuis leur enfance. Nous avons constaté qu'il faut mettre les enfants en contact fréquent avec la tradition et ainsi permettre une transmission intergénérationnelle.

Une autre chose qui m'a frappée en écoutant madame Audet, c'est l'appartenance. Ce sont ses danses à elle, presque son Île d'Orléans. On l'a beaucoup rencontré ce phénomène-là. Il témoigne de l'appropriation par les gens de leur répertoire dans une trajectoire qui se vit d'une certaine manière. Mais pour l'enfant qui était dans le film et qui regarde ça, il va avoir un autre vécu, il va vivre dans un autre contexte et traduire ce qu'il 
a reçu comme information, ce qu'il a observé comme le plaisir des gens qui dansent, et le traduire dans sa réalité plus tard. Des fusions, il y en a toujours eu, comme des choix esthétiques, acoustiques comme électriques. Pour moi, que des gens fassent des fusions ou non, l'important est qu'il se passe quelque chose. Je préfère qu'il y ait des fusions, mais qu'il y ait justement des ethnologues et des archives, des références, des portraits d'une époque à laquelle on peut se référer si on veut savoir, entre guillemets, la vérité. De toute façon, la vérité va évoluer, changer avec les gens. Toute expression est importante parce que c'est ce qui fait que c'est vivant, aujourd'hui, maintenant.

LouIs WaUthier. J'aime bien ce que tu dis, Danielle. Ça répond en partie à la question et ça va dans le sens dont Tess parlait tout à l'heure. Ils vont peut-être avoir oublié des choses, mais ils vont l'exprimer sur scène ou dans leur communauté à leur façon.

Tout à l'heure, on se demandait : où vont-ils chercher les sources ? Des associations comme celle-ci ont le mandat de justement conserver. Si on n'avait pas des gens qui ont fait de la recherche, on ne l'aurait jamais vu ce film-là ; on ne pourrait pas dire que le quadrille, ou le lancier, ou le saratoga, en 1976, au Château Bélair, s'est dansé comme ça. Un jour, $\mathrm{j}$ 'assistais à une conférence de Simonne Voyer. Après la conférence, elle me dit : « Tu sais, lorsque j'ai pris en note le cotillon de Baie-SainteCatherine à Sainte-Rose-du-Nord, en 1964, je peux te dire qu'en 1964 il se dansait comme ça. Est-ce qu'il se danse comme ça en 1994 ? Je n'en ai aucune idée. Il a changé, il a évolué, selon ce que les gens ont bien voulu en faire ». Même si on n'y fait pas toujours référence quand on calle une danse, c'est important d'en connaitre la source.

FranCINE ReEves. J'ai une question à poser à propos des Cailloux qui ont chanté au moment où je suis partie en France. En 1966, ils étaient très actifs. Quelle influence ont-ils eue ? Raoul Roy était également très actif à ce moment-là.

La question identitaire a été importante dans les années 1970. Dans les villes, le folklore a été lié à l'identité québécoise. Beaucoup de jeunes se sont alors intéressés à cette musique et à ce répertoire. Je sais qu'il y a eu un essoufflement après l'échec référendaire. Beaucoup ont lâché à ce moment-là. Il semble y avoir eu comme un lien absolument obligatoire entre l'indépendance québécoise et le répertoire. J'étais en France à ce moment-là et j'ai commencé, tout à fait par hasard, à animer des soirées de danses québécoises, en même temps, sans savoir qu'il y avait ce renouveau ici. Il y avait également ce même renouveau en France, ce qui m'a probablement supportée aussi. C'était peut-être mondial, en fait. Je 
me demande quelle influence ont eue, par exemple les Cailloux, qui ont popularisé un répertoire qui se chante encore.

RoBert Bouthillier. En tout et pour tout, les Cailloux ont fait quatre disques, ce qui veut dire grosso-modo 40,45 chansons qui ont circulé, dont peutêtre trois, quatre ont été des tubes comme Au chant de l'alouette et Mon canot d'écorce. Je pense qu'ils ont eu essentiellement une influence sur les jeunes urbains. Ils semblent avoir eu très peu d'influence auprès de gens que j'ai pu interroger dans les années 1970 dont les références étaient beaucoup plus des vedettes comme Ovila Légaré et la Bolduc, qui ont eu une influence certaine sur une tradition générale, collective. L'influence des Cailloux reste extrêmement marginale et dans un milieu intello, boîte à chansons de l'époque.

Francine ReEves. Ce sont quand même les milieux urbains qui ont effectué au plus fort cette relation avec l'identité québécoise.

ROBERT BOUTHILLIER. Dix ans plus tard.

Francine Reeves. Oui, mais ça venait de quelque part. Ce n'est pas sorti de nulle part.

RoBert BOuthillier. Je laisse à d'autres le soin de répondre.

Monique Jutras. L'histoire et mes études à l'université m'ont montré que nationalisme égale intérêt envers le folklore. Cela s'est vu dans différents pays et à différentes époques. Pour moi, le revival du folklore au Québec, ç'a duré jusqu'à l'arrivée du Parti québécois au pouvoir. Dès que le PQ a été au pouvoir, et même avant le référendum en 1980, ç'a été comme acquis et l'intérêt a commencé à baisser. Le $\mathrm{PQ}$ au pouvoir allait s'occuper de tout, on n'avait plus besoin de se prononcer.

FRANCINE REEVES. Je pense qu'effectivement c'étaient des phénomènes urbains et dans les campagnes, les gens continuaient, en tous les cas pendant un certain temps, à pratiquer ces répertoires sans se poser les mêmes questions ou procéder à la même identification.

Monique Jutras. Ç'a eu un effet parce que, quand je regarde les Robert Charlebois, Gilles Vigneault, Louise Forestier, nos vedettes québécoises qui faisaient des disques et qui tournaient à la radio, tout le monde les connaissait, à la ville comme à la campagne. Dans l'esprit populaire, ces gens faisaient du folklore. Plus personne ne savait vraiment ce que c'était. Tout était folklore.

Donald DeschêNes. Normand veut réagir.

Normand Legault. En discutant, on se rapproche de ce que sont les voies de la tradition. Tantôt on est spectateur, tantôt on est en situation 
d'apprentissage. On peut être spectateur en regardant un film de madame Audet trente ans après. On peut être spectateur en écoutant les Cailloux ou en regardant à la télévision Le Ranch à Wellie ou Soirée canadienne. Ce sont tous des outils qui nous rentrent dans la tête et qui forment notre connaissance du folklore ou du patrimoine. C'est à partir du vécu de chacun, il n'y en a pas un qui est pareil. Faut avoir dansé pendant peutêtre dix ans au Château Bélair et avoir le vécu de ces gens-là, ou avec la Parenté au pavillon Pollack à l'université Laval. Ça fait partie de l'apprentissage. Mais, à un moment donné, quand on a assez d'outils, on devient interprète et acteur. Ce sont les autres qui sont alors acteurs de notre action. Il y a passage. On peut rester encore spectateur, il y a toujours des nouveautés qu'on ajoute dans le bagage. « Envoye dans ma voiture ", comme le dit la chanson. Mais quand on devient acteur, on pige dans ces modèles pour créer. Je fais de la danse, j'écris des choses, je pige dans tout ça. Je n'élimine pas cela parce que ça fait swing des années 1950. Ça fait partie du même pot. Comme acteur, je suis amené dans mon travail à interpréter; je travaille avec ce que j'ai en moi et pouf, ça sort. Il y a cette notion de spectateur/acteur. On est toujours un peu entre les deux, mais, avec le temps, on devient de plus en plus interprète et acteur, et une référence pour d'autres. Les voies de la tradition, c'est également ce passage.

Pierre Chartrand. Deux petits points. On sait qu'en Bretagne, au pays Basque, au Québec, là où il y avait des revendications nationalistes, le folklore, les pratiques du genre revivaliste ont été très portées par ce mouvement. Je suis d'accord, mais je vais quand même mettre un bémol parce qu'on sait aussi qu'une certaine intelligentsia québécoise, politique entre autres, était contre.

Je me rappellerai toujours un fameux coquetel, aux environs de 1982, avec Clément Richard, alors ministre de la Culture. Je m'en allais faire une conférence à Atlanta, et, comme d'habitude, je n'avais pas assez de financement. Il me dit : « Vous comprenez, monsieur Chartrand, ce n'est vraiment pas dans la politique du PQ d'exporter l'idée d'un Québec traditionnel ». Le PQ voulait montrer un Québec moderne. Vingt ans après, la ministre Beaudoin ne voulait pas de folklore au Printemps $d u$ Québec à Paris. J'ai envoyé ma lettre au Devoir et le sous-ministre m'a appelé pour me dire: "Ce n'est pas l'opinion de madame Louise Beaudoin, mais de sa porte-parole ». Elle la paie, sa porte-parole. Et puis Robert Lepage qui dit qu'il faut montrer le Québec moderne et laisser tomber les ceintures fléchées, Maria Chapdelaine et les rigodons. Donc, 
nationalisme rime avec folklore, oui, mais avec des bémols. C'était le premier point.

Plusieurs d'entre nous avons appris et sommes devenus des passeurs de savoirs. Je trouve qu'il y a une certaine continuité entre les différentes périodes de renouveau et c'est souvent en réaction à une culture de masse. On retrouve ça chez les jeunes aujourd'hui comme on le voyait dans les années 1970. À cette époque, Marcuse parlait de réagir à la culture de masse. Aujourd'hui, on entend un peu le même discours par rapport à la mondialisation. J'y vois une continuité. La pratique, spectaculaire ou non spectaculaire, est souvent en réaction au rouleau compresseur de la culture de masse - télé, radio - , aux produits tout cuits. Notre façon d'être acteur, évidemment, c'est une réaction parce que, si on danse, si on joue, on n'est pas passif.

MoNIQUE JUTRAS. Je reviens encore avec mon idée : la scène c'est une chose, la tradition c'en est une autre. Et c'est le choix des artistes, c'est leur privilège de faire ce qu'ils veulent avec la tradition. Il y en a qui peuvent décider de prendre la tangente puriste et ne pas changer les chansons, prendre même la gestuelle des chanteurs traditionnels, chanter a cappella et les reproduire sur scène. D'autres peuvent décider de faire des fusions.

Aujourd'hui, au moment où on n'a plus de transmission orale, de génération en génération, le rôle des ethnologues est extrêmement important. Comme le disait Danielle, il faut qu'on puisse toujours avoir accès à des documents d'archives. C'est important.

Le problème, c'est toujours le grand public, la masse, la collectivité québécoise. On est toujours au même point, on n'est pas fier de notre folklore. Collectivement, ce n'est pas valorisé. On se bat encore aujourd'hui pour le faire reconnaître. Dans les pays d'Europe de l'Est, en Amérique Latine, les gens ne sont pas honteux. Ici, il faut souvent s'excuser de faire une chanson de folklore. À la SPDTQ, on invite des chanteurs, des conteurs traditionnels qui ont peut-être moins le sens de la scène, mais qui sont plus authentiques, d'une tradition plus pure. J'adore ça. Mais est-ce que ces événements disent quelque chose au grand public ? On a toujours un choix à faire. Il m'apparaît important de poursuivre la sensibilisation de la population québécoise. C'est important peut-être de leur dire la vérité, notre vérité.

Robert Bouthillier. J'avoue que j'ai bien de la misère avec les mots authenticité et purisme. Ça m'énerve, ces mots-là. Pour moi, ça n'existe pas ; ça ne sert à rien d'essayer de nommer quelque chose qui n'existe pas. Louis disait qu'en 1964, à Sainte-Rose-du-Nord, ça se dansait de 
même. C'est objectif, clair, net, concis. Ce que nous, ethnologues c'est un autre mot que je n'aime pas beaucoup -, appelons la source, nous avons effectivement la responsabilité de la créer et de la rendre accessible à titre d'information et, éventuellement, de formation, sachant que c'est totalement insuffisant pour qu'une culture, quelle qu'elle soit, puisse se perpétuer d'une façon ou d'une autre, ou puisse traverser, en même temps que le temps, les esprits, les cerveaux, les communautés, la pratique collective. Donc, on a un sacré boulot à faire à ce niveau-là.

Je suis d'accord avec Monique sur un point, c'est que la scène est absolument insuffisante à faire cette médiation-là entre des choses qui, effectivement, appartiennent à d'autres contextes, à d'autres univers, à d'autres types de fonctionnement culturel, et la société d'aujourd'hui. Elle ne se fout pas des choses, elle ne sait pas qu'il existe des choses, c'est pas pareil. Nous, on a une responsabilité d'information. Mais j'avoue que c'est dur d'informer six millions ou six milliards de personnes. J'ai commencé à penser que je n'aurais pas le temps de le faire et j'essaie de travailler autrement. J'ai bien aimé l'image de Normand : on est au début des éponges, on s'imprègne de choses, on participe à un univers. Soit qu'on naît dans un village, qu'on naît dans une famille de chanteurs, qu'on a appris des affaires de la grand-mère, du grand-oncle, et on les sait par cœur. Des gens comme Gaston Lepage, c'est exactement ça. Eux, ils ne se posent pas de questions, ils ne se coupent pas les cheveux en quatre en se demandant qui ils sont et pourquoi ils font les choses. Ils les font. Pour différentes raisons, je n'ai pas l'héritage familial de Gaston, mais j'en ai plein d'autres. C'est vrai que je suis une somme de choses, autant de mon travail de chercheur que de mon travail d'humain en contact avec les gens, que de mon travail de voyageur qui a vu et d'oreille qui a entendu plein de choses. On est tous une espèce de synthèse individuelle et pas seulement héritier de la communauté dans laquelle on est inscrit. Et c'est comme ça, maintenant, qu'on doit travailler. Parce que je n'ai pas l'impression d'avoir la vérité avec un grand $V$. Je ne suis finalement qu'un état d'un savoir que j'ai systématisé ou organisé à ma façon, comme d'autres le font à leur façon. C'est la synthèse de tout ça finalement qui est l'espèce de ligne médiatrice qui nous relie à un empremier, un avant, à des anciens qu'on a enregistrés, des mémoires qu'on a fait surgir, des esthétiques qu'on est allé voir et toucher, et qui nous ont frappés et formés aussi. Et la scène est insuffisante ; faut travailler autrement.

Je reprends l'exemple des enfants de Danielle. Quand on arrête d'essayer de changer le monde dans son entier, on travaille au niveau de communautés peut-être un peu plus restreintes. On ne travaille pas en démonstration, on revient quasiment à une logique du quotidien ou de 
l'appartenance, mais de l'appartenance à un milieu où chacun se reconnaît. Le Château Bélair, c'était ça. J'ai quasiment le goût de revenir au sacré de Normand parce que c'est vrai que cette chose-là, ça devient l'élément. C'est vrai qu'on boucle des boucles. J'ai la chance de vivre dans un milieu où cette convivialité se vit. Elle ne se vit pas par héritage obligé, elle se vit par choix. Aujourd'hui, on est à l'époque de la culture choisie. Je décide que je vais à telle veillée, je décide que je vais à ce repas chanté qu'on organise avec cinquante personnes qui ne sont pas les voisins du village, mais des gens qui viennent de quelques kilomètres à côté. Mais on va créer tout de même dans ces nouveaux contextes, de l'appartenance, du partage, l'impression qu'on est ensemble et qu'ensemble on fait, on vit quelque chose. On ne montre pas, on ne démontre pas, on ne donne pas un spectacle, on fait, tout simplement. Quand ça se fait dans une verticalité de générations, c'est là qu'on a gagné. C'est là que, dans ce milieu d'appartenance, le grand village ouvert, les gens viennent ou ne viennent pas, selon; ils ont le choix. Mine de rien, la convivialité l'emporte très souvent sur l'aspect peut-être un peu rébarbatif des choses non spectaculaires. Les gens ont du plaisir à revenir et c'est comme ça que ça se fait. Quand ce sont des enfants de cinq, six ans qui se mettent dans le rond et qui dansent avec la grand-mère de 70 ans, c'est gagné. Là, c'est sûr que pour eux, ça ne sera pas un lieu de culture du spectacle, vaguement identitaire, idéologique, politique, nationaliste. On travaille beaucoup plus à l'appartenance, à quelque chose qui commence à ressembler à une communauté qu'on choisit, qu'on se fait. Si on a des avenues à explorer pour les voies, v-o-i-e-s, c'est vers ça. Et là-dedans, les voix, v-o-i-x, ont leur place. Les gens vont se donner le droit de chanter comme ils le savent et non pas comme on leur dit qu'il faudrait qu'ils chantent. Encore une fois, ce sont des avenues gagnantes, sachant qu'on travaille au niveau de quelques centaines et non pas de millions de personnes. J'ai passé l'âge de m'attaquer à des gâteaux trop gros pour être digérés. À mon sens, c'est ce qu'on peut appeler une tradition. Une tradition, je vous rappelle le mot, ça traverse. Et même au XXI siècle, ça se peut.

Donald DeschêNEs. En écoutant Robert, je me suis tout à coup retrouvé dans les cours de notre ami Du Berger, avec des mots importants : communauté, plaisir, sacré. On revient toujours à l'essentiel.

Danielle Martineau. Monique est inquiète de savoir qu'est-ce que le grand public sait ou ne sait pas. Il y a peut-être cinquante ans, le grand public n'existait pour ainsi dire pas, dans le sens que l'entendent les médias. L'action était plus près, familiale, communautaire, villageoise. De la 
même manière, les arts de la scène existaient, mais l'industrie n'était pas organisée comme elle l'est aujourd'hui. Dans nos enquêtes, à Lisan et moi, sur la transmission, ça se résume au plaisir de se rencontrer le plus possible, sans équipement et grosse organisation. Ce qui compte, c'est que chacun est invité à participer, à préparer quelque chose à faire d'inclusif. Si on veut permettre aux gens d'avoir des occasions comme ça, il faut se baser sur ce qu'ils nous ont transmis comme sens de la communauté.

Donald DeschêNes. La communauté signifie que chacun est spectateur, mais chacun est également participant. Monsieur Wauthier, vous vouliez réagir?

LOUIS WAUTHIER. Suite à ce que tu disais, Monique, tout à l'heure, ton questionnement m'a amené à me poser des questions, dont celle de la honte de notre folklore. J'ai souvent entendu des professeurs de danses ethniques, entres autres, qui ne comprenaient pas pourquoi on est si ouvert à la culture des autres, des danses japonaises, tchèques, yougoslaves, et qu'on ait une si pauvre idée de sa propre culture. Combien je reçois de téléphones de commissions scolaires qui veulent avoir des cours pour les petits, mais uniquement en parascolaire, une heure ici et là. Ce n'est pas intégré.

Normand Legault. Ici, il y a des gens qui sont dans le milieu depuis trente, quarante ans, des gens qui sont nouveaux. Je trouve qu'on est rendu des experts en isolation. Pour contrer le fait que c'est peu connu et pas tellement apprécié, on s'est surspécialisé et on ne fait que trop d'une seule affaire. On fait de la gigue, de la danse, on participe à des festivals, on en parle pendant quatre à cinq jours et après, plus rien. Ça veut dire qu'on ne rejoint pas la communauté. La sphère de la continuité, on n'est pas dedans. Dans la vie courante, le samedi soir, je vais manger du sushi, boire du saké, faire des choses qui n'ont rien à voir avec la tradition. Si on fait de la danse, il faut que ça soit comme ça se faisait, de la gigue comme ça se faisait. Si on faisait une veillée de danse traditionnelle au pavillon Pollack à l'université Laval, nous aurions l'air de Martiens. Maintenant, il n'y a que des initiés qui savent apprécier ce que nous faisons. À la radio, on n'entend plus de la musique traditionnelle que pendant les fêtes, jusqu'à en être saturés. Même par notre action, on isole des phénomènes, on les sort de leur contexte. Regardez le film avec madame Audet, le contexte était complet. À cette époque, les gens se retrouvaient. Ça venait de l'île, de partout. On dansait sans qu'on ait besoin de caller les danses. La continuité était là, simplement. Ce n'était pas plus extraordinaire que cela. Maintenant, on fait une soirée de danses québécoises ; on loue un gymnase, une estrade, une palette de bois, un 
système de son, on se plaint si les moniteurs sont trop loin. Il faut qu'on organise la publicité et ça ne marche pas, il n'y a pas de monde, le plancher est dur. Il n'y a plus de plaisir. On essaie tant bien que mal de faire survivre la tradition dans des contextes qui n'ont plus rapport avec l'objet même du plaisir. On invente des communautés, des gens qui dansent ensemble et qui ne se connaissent pas. Je pense qu'on est trop missionnaire. C'est ce qui fait qu'on continue, mais peut-être qu'on échoue ou qu'on n'a pas trouvé la bonne façon de faire. Il y a là un questionnement que je n'ai pas encore compris. On est rendu des experts de pratiques intensives en milieu professionnel, mais, dans la vie quotidienne, on n'est presque plus là-dedans.

LISAN HUBERT. Je voulais parler de l'intérêt qu'ont ou n'ont pas les gens pour la musique, la chanson, la danse traditionnelle. Dans nos enquêtes dans Lanaudière, nous avons rencontré des gens qui ont été élevés dans la tradition. Ils étaient cinq enfants, les parents les mettaient sur une table et les faisaient chanter et danser. Ils ont été élevés dans la musique traditionnelle pure. Mais eux, les enfants, ce n'est pas ça qu'ils ont aimé. $\grave{A}$ quinze, seize ans, ils ont appris Elvis Presley, ils ont tous fait de la musique. Aujourd'hui, ils font de la musique western, une musique bien proche de la musique traditionnelle. Le fait qu'on soit exposé au folklore ne fera pas qu'on va nécessairement s'y intéresser. Il faut faire entendre aux enfants un large éventail de musiques. Certains vont rester ouverts à la musique traditionnelle et s'y intéresser.

Monique Jutras. J'ai l'impression que l'époque des grands rassemblements collectifs au Québec est révolue. Dans les années 1970, tout le Québec vibrait au même rythme. Aujourd'hui, les choses se vivent beaucoup plus en petits groupes, en cellules.

Cette discussion m'amène à trois constats par rapport aux voies que la tradition a prises. Premièrement, le niveau de la qualité de ce qui se fait sur scène a continuellement augmenté ces trente dernières années. On a des jeunes qui s'intéressent au folklore, qui sont passionnés et qui ont des formations musicales. On trouve d'excellents joueurs de violon et d'accordéon, beaucoup plus qu'il y a trente ans. Ils savent se présenter sur scène.

Mon deuxième constat : dans les années 1970 et peut-être même dans les années 1980, Robert était vraiment un missionnaire de l'ethnologie. Avant de partir pour la France, il nous avait organisés pour qu'on continue, pour qu'il se passe quelque chose ; il ne fallait pas laisser tomber. Je vois qu'aujourd'hui le folklore est toujours un milieu un peu marginal, mais en retournant chez moi, à Montréal, il y a quelque temps, 
j'ai découvert un milieu plus grand que celui de Québec. Les veillées de la SPDTQ regroupent deux ou trois cents personnes qui peuvent aller danser. La semaine passée, il y avait deux événements de folklore en même temps : une danse et le jam session Les portes d'en arrière. Donc, il y a des choses qui se passent et il y a des gens pour qui cela devient leur quotidien. Même chose à l'Association québécoise des loisirs folkloriques (AQLF). Ce sont des lieux d'apprentissage de la musique traditionnelle, violon, harmonica, accordéon et guitare. Aujourd'hui, on vit peut-être nos choses en plus petits groupes. Il en sortira peut-être quelque chose dans dix ou quinze ans.

Un dernier commentaire : si la culture canadienne-française a continué, c'est parce qu'on s'est refermé sur nous-mêmes après la conquête anglaise. Si le repli sur soi est négatif dans un sens, il a été important pour la conservation de la culture traditionnelle. Par ailleurs, nous sommes ouverts au Québec, il n'y a qu'à voir notre attitude envers les immigrants. Nous acceptons qu'ils portent leur arme ou le voile à l'école. Mais l'affirmation de notre culture, où est-elle ? Comme si tout ce que font les autres était meilleur. Donc, prudence.

Donald Deschênes. Merci Monique. Un dernier commentaire?

Pierre Chartrand. Nous avons souvent un discours misérabiliste. Nous sommes marginaux et nous devons l'être. Par rapport à la question ethnique, je fais des spectacles de gigue. Une des jeunes danseuses, une fille de la République centrafricaine, qui est au Québec depuis quelques années, trippe sur la gigue. Il faut se poser la question : pourquoi n'y a$\mathrm{t}$-il pas plus souvent, surtout dans des villes comme Montréal et Québec où il y a des communautés ethniques, des gens des communautés ethniques à nos activités?

Donald Deschênes. Monsieur Du Berger, êtes-vous en mesure de résumer?

JeAN Du Berger. Non! Porter des conclusions, j'en suis incapable. Il y a quand même une chose qui m'a frappé, c'est que, pour la plupart d'entre nous, on s'est situé dans une activité de sauvegarde. C'est-à-dire : ça va mourir, ça disparaît, il faut se dépêcher à le sauver. Hâtons-nous de recueillir les histoires du peuple. Ça remonte au temps de Boas, il fallait sauver la culture amérindienne. Il y a un article de Michel De Certeau sur la beauté du mort qui dit : quand les gens meurent, on se dit : c'est très très beau. C'est le travail que nous avons fait pendant longtemps : sauvegarder. Une autre chose aussi, c'était l'authenticité. Je me souviendrai toujours, certains de mes collègues à l'université Laval, qui étaient là : est-ce que c'est vraiment authentique ? Peut-être que cette 
chanson-là est littéraire ! C'était la grande stigmate, c'était littéraire, même si tout le monde la chantait. Pourtant! Il faut faire bien attention. La pureté, l'authenticité, en fait, c'était la recherche d'un monde disparu, d'un monde qui n'a jamais existé.

Ce qui m'a frappé chez madame Audet, c'est quand elle dit : " Ça, c'est à nous autres. Il ne faut pas donner ça aux autres ». Ernest Fradette, quand il est venu à mon bureau, il n'avait pas l'impression de se situer dans Aarne-Thompson, dans le Types Index. Non, ses contes sont des affaires qui sont arrivées à côté de chez eux. Ça lui appartient. Un jour, quelqu'un est allé dans un des spectacles de Barbeau. Quelqu'un contait un conte en avant. Il dit : « Aïe, de quel droit contez-vous mon conte ? » Il pensait que c'était à lui.

Alors, on est devant des cultures qui étaient très très proches des familles. Ma première découverte en enquête, c'était avec les Juneau à Shawinigan. Cette famille-là, qui ne chantait plus, le faisait parce que moi j'y allais pour aller recueillir des faits de folklore. J'ai revu de ses enfants qui étudiaient en droit à Laval, qui venaient chercher mes enregistrements pour se souvenir de ce qu'ils avaient entendu quand ils étaient jeunes. Le référendum, nous autres à Laval, a fait chuter le recrutement des étudiants. C'est là qu'a commencé la dégringolade.

Maintenant, il y a la toile électronique. Les groupes d'appartenance se forment autour des clavardages. Même les contes se transmettent par là. Les rumeurs font le tour de la terre en peu de temps. Alors, il y a une autre transmission. Ça va cent fois plus vite qu'autrefois. Mais il y en a encore de la transmission. Ça n'arrête pas de parler, ce sont les rumeurs urbaines, les légendes. Il y a des transformations dans le domaine du conte que je connais un peu. Ce sont, par exemple, les contes urbains. Il y a une activité langagière. Ce n'est plus une transmission directe. On a retrouvé des choses qui circulent sur internet, des conteurs les reprennent après. Alors, il y a des nouvelles voies de transmission. Et c'est vrai, ça aussi, ce à quoi vous avez fait allusion, il se forme des petits groupes d'appartenance, tout à coup on se retrouve dans les veillées. Les gens se retrouvent là-dedans.

Je finis sur une confidence parce que j'ai trouvé un nouvel usage du conte. Depuis la retraite, je suis devenu bénévole pour la Maison MichelSarrazin, destinée aux soins palliatifs pour cancéreux. Je me sers des contes avec les malades, des contes pour guérir. En fait, ça guérit l'âme. Je fais les récits de vie des gens. C'est très surprenant, ça reprend la trame des contes traditionnels. C'est Ti-Jean qui part, qui fait sa vie. Le dernier monstre qu'il a rencontré, c'est le cancer. Mais il y a toujours l'attente de quelque chose, comme le conte des deux petites grenouilles 
qui viennent leur donner de l'espoir pour se faire aller les pattes. Comme le dit Vigneault, «Les gens de mon pays, ce sont gens de paroles, des gens de causerie ». Mais c'est un milieu très spécial, c'est le dernier chant et c'est quelque chose de très beau.

Pour finir, il y a un bûcheron, un homme d'une quarantaine d'années qui a le cancer du palais, les deux yeux cautérisés, plus de nez. Pour ceux qui fument encore, je peux vous dire que le cancer du palais, ce n'est pas beau à voir. Tout le monde en avait peur. La femme médecin qui était là me dit : «Va donc le voir ». J'arrive et je lui dis : « Vous êtes bûcheron ? - Oui, monsieur. » J'ai dit : « J'ai déjà vu des photographies de grands traîneaux chargés de billots qui dépassaient les arbres, tirés par deux chevaux. Comment faisiez-vous ? » Là, il faut reprendre la parole de Pierre Perreault : il m'a pris la parole puis il l'a gardée pendant trois heures. Pendant ces trois heures, cet homme qui souffrait, il n'a pas pris une entredose. Ses douleurs étaient supprimées. À la fin, il m'a donné la main et il a dit : « C'est le plus bel après-midi de ma vie ! » Il est mort le lendemain. Voyez-vous ? La parole l'avait guéri pendant quelque temps. Ce que les conteurs faisaient, ils guérissaient leur communauté, ils apportaient la parole. Ce n'est pas mort, ça continue. Il s'agit pour nous autres de la reprendre la parole, de la porter. Merci.

Donald DeschêNes. Merci Jean. Merci à tous les participants. 